\title{
RAl1 Gene
}

National Cancer Institute

\section{Source}

National Cancer Institute. RA/1 Gene. NCI Thesaurus. Code C75453.

This gene is involved in transcription, development and behavior. 